Portland State University

PDXScholar

2-1-1971

\title{
Dielectric Polarization and Alignment and the Structure of Polar Fluids
}

John D. Ramshaw

Portland State University, jdramshaw@yahoo.com

D. W. Schaefer

Massachusetts Institute of Technology

John S. Waugh

Massachusetts Institute of Technology

J. M. Deutsch

Princeton University

Follow this and additional works at: https://pdxscholar.library.pdx.edu/phy_fac

Part of the Physics Commons

Let us know how access to this document benefits you.

\section{Citation Details}

J.D. Ramshaw, D.W. Schaefer, J.S. Waugh, and J.M. Deutch, "Dielectric Polarization and Alignment and the Structure of Polar Fluids," J. Chem. Phys. 54, 1239 (1971)

This Article is brought to you for free and open access. It has been accepted for inclusion in Physics Faculty Publications and Presentations by an authorized administrator of PDXScholar. Please contact us if we can make this document more accessible: pdxscholar@pdx.edu. 


\title{
Dielectric Polarization and Alignment and the Structure of Polar Fluids*
}

\author{
J. D. Ramshaw, $\dagger$ D. W. Schaefer, $\ddagger$ and J. S. Wavgh \\ Department of Chemistry, Massachusetts Institute of Technology, Cambridge, Massachusetts 02139 \\ AND \\ J. M. Deutch $\$$ \\ Department of Chemistry, Princeton University, Princeton, New Jersey 08540
}

(Received 19 December 1969)

\begin{abstract}
An analysis is made of the information about the structure of dense polar fluids which resides in the dielectric constant, the Kerr constant, and the nuclear magnetic resonance (NMR) quadratic electric field effect. The inadequacy of the "local-field" model for liquids is discussed. The existence of a nonzero molecular hyperpolarizability is shown to destroy an equivalence which would otherwise exist between the Kerr and NMR experiments, and can easily account for apparent discrepancies between the reported Kerr and NMR data for nitrobenzene and nitromethane. A method is presented for removing dielectric boundary effects from statistical averages, so that the averages can be computed locally.
\end{abstract}

\section{INTRODUCTION}

It has long been realized that measurements of the dielectric constant provide a means of investigating intermolecular forces and the local order which these forces produce in dense polar fluids. In order to interpret the dielectric constant on the basis of the purely local microscopic structure of the fluid (i.e., the shortrange correlations between a representative molecule and its nearest neighbors) it is necessary to separate the local-order contributions from the apparently macroscopic contribution of shape-dependent boundary effects. Kirkwood ${ }^{1}$ developed an approximate method of effecting this separation.

The dielectric constant, however, is not the only observable quantity associated with the interaction between a polar fluid and an applied electric field. Two other "electric-field effects" of interest are the Kerr effect and the quadratic electric-field effect in NMR. One is led to wonder whether an extension or modification of Kirkwood's approach would make it possible to obtain additional information about the local structure of fluids from these experiments or from other electric-field experiments. The present paper provides an affirmative answer to this question, and is devoted to a detailed examination of the dielectric, Kerr, and NMR experiments from a unified viewpoint. Our treatment reveals explicitly the interrelation between these experiments and the different kinds of structural information which can be obtained from them.

We begin by considering a spherical sample in vacuum. In order to eliminate the boundary effects associated with this sample geometry, we will later transform to the case of a spherical sample immersed in an infinite medium of the same sample material. In either case, we have isotropy in the absence of the applied field. We shall be concerned with quantities of the form $\left\langle P_{n}\left(\cos \theta_{1}\right)\right\rangle_{E}$, where $\theta_{1}$ is the angle between the permanent dipole moment of a representative molecule 1 and the (uniform) externally applied field E, $P_{n}(z)$ is the $n$th Legendre polynomial, ${ }^{2}$ and the brackets $\langle\cdots\rangle_{\boldsymbol{E}}$ denote an average over the positions and orientations $\left(\mathbf{R}^{N}, \boldsymbol{\omega}^{N}\right)$ of all $N$ molecules in the sample, weighted by the Boltzmann factor appropriate to equilibrium in the presence of $\mathbf{E}$. It is clear that these quantities provide a description of how single molecules are aligned by the field. ${ }^{3}$ We are particularly interested in $\left\langle\cos \theta_{1}\right\rangle_{E}$ and $\left\langle P_{2}\left(\cos \theta_{1}\right)\right\rangle_{E}$, which we refer to as the polarization and alignment, respectively. We will presently show that the dielectric constant can be regarded as a measure of the polarization, while the NMR electric-field effect is a measure of the alignment and the Kerr effect measures a linear combination of the two.

Since laboratory electric fields are weak in comparison to typical molecular fields, we are normally interested in the low-field limit of $\left\langle P_{n}\left(\cos \theta_{1}\right)\right\rangle_{E}$; that is, in the first nonzero term in a Taylor series expansion about $\mathbf{E}=\mathbf{0}$. This limit is implicit throughout the paper unless otherwise stated. We therefore specifically exclude saturation phenomena from our discussion, although their inclusion would in principle present no difficulty. By exploiting the rotational transformation properties of a tensor of arbitrary rank $^{2}$ and making use of a spherical average (see Sec. VI), one can show, in general, that $\left\langle P_{n}\left(\cos \theta_{1}\right)\right\rangle_{E}$ is of order $E^{n}$ in the low-field limit. Thus the polarization and alignment are linear and quadratic in the field, respectively.

A word about electric fields is in order. We denote by $\mathbf{E}$ the uniform externally applied electric field, whose sources are external to the sample material and are assumed fixed. It is the field $\mathbf{E}$ with which the sample must be considered to interact. The macroscopic Maxwell electric field, obtained by solving the macroscopic Maxwell equations subject to the usual electrostatic boundary conditions, is denoted by $E_{m}$. For a sphere in vacuum, it is well known that $\mathbf{E}_{m}=3 \mathbf{E} /(\epsilon+2)$, where $\epsilon$ is the dielectric constant.

For simplicity, we will limit our discussion to molecules of axial symmetry. The molecular configuration of the sample, over which statistical averages are to be performed, is considered to consist only of the set 
$\left(\mathbf{R}^{N}, \boldsymbol{\omega}^{N}\right)$ of all molecular positions and orientations; all other degrees of freedom, including molecular vibration, are considered to be "internal" molecular coordinates. All molecular parameters, such as the permanent dipole moment and the polarizability, are implicitly regarded as the appropriate averages over these internal coordinates.

In Appendix A we review for convenience the conventional description of the polarization of an isolated molecule by a uniform electric field.

\section{THE DIELECTRIC CONSTANT}

In order to reveal the dependence of the dielectric constant on the polarization, we consider the average net dipole moment per unit volume, $\mathbf{P}$. In general, $\mathbf{P}$ is a function of position $\mathbf{P}(\mathbf{r})$, but for a sphere in vacuum both $\mathbf{P}$ and $\mathbf{E}_{m}$ are uniform within the sample. Then

$$
\mathbf{P}=V^{-1}\langle\mathbf{M}\rangle_{E}=V^{-1}\left\langle\sum_{k} \boldsymbol{\mu}_{k}\right\rangle_{E}=\rho\left\langle\boldsymbol{\mu}_{1}\right\rangle_{E}
$$

where $\mathbf{M}$ is the instantaneous total electric dipole moment of the sample, produced by a particular configuration of the molecules, $\boldsymbol{\mu}_{k}$ is the dipole moment of molecule $k$, and $\rho$ is the number density. The constitutive relations of electrostatics $\left(\mathbf{D}=\mathbf{\epsilon E}_{m}=\mathbf{E}_{m}+\right.$ $4 \pi \mathrm{P}$ ) and the spherical geometry then imply that

$$
(\epsilon-1) /(\epsilon+2)[3 /(4 \pi \rho)]=\left\langle\mu_{1} \cdot \mathbf{e}_{\mid i}\right\rangle_{E} E^{-1},
$$

where $\mathbf{e}_{\|}$is a unit vector in the direction of $\mathbf{E}$. The moment $\mu_{1}$ is the sum of the permanent moment $\boldsymbol{\mu}_{1}{ }^{0}$ (i.e., the moment of the isolated molecule in zero field) and the moment induced by the external field and by interaction with all the other molecules; it therefore has to be written as $\boldsymbol{\mu}_{\mathbf{1}}(\tau, \mathbf{E})$, where $\tau$ is a short-hand notation for the molecular configuration $\left(\mathbf{R}^{N}, \boldsymbol{\omega}^{N}\right)$. In the low-field limit, we may write

$$
\begin{aligned}
\left\langle\boldsymbol{\mu}_{1} \cdot \mathbf{e}_{\| \mid}\right\rangle_{E}=\left\langle\boldsymbol{\mu}_{1}(\tau, 0)\right. & \left.\cdot \mathbf{e}_{\| \mid}\right\rangle_{E} \\
& +\left\langle\left[\partial \boldsymbol{\mu}_{1}(\tau, \mathbf{E}) / \partial \mathbf{E}\right]_{E=0}: \mathbf{e}_{\|} \mathbf{e}_{\| \mid}\right\rangle_{0} E .
\end{aligned}
$$

Let $\left(\mathbf{e}_{1}, \mathbf{e}_{2}, \mathbf{e}_{3}\right)$ be a set of orthogonal unit vectors fixed with respect to molecule 1 , with $e_{1}$ taken along the symmetry axis (permanent moment direction). Then

$$
\left\langle\mu_{1}(\tau, 0) \cdot \mathbf{e}_{\| 1}\right\rangle_{E}=\sum_{i}\left\langle\left[\mu_{1}(\tau, 0) \cdot \mathbf{e}_{i}\right]\left(\mathbf{e}_{\| \mid} \cdot \mathbf{e}_{i}\right)\right\rangle_{E}
$$

We now neglect fluctuations of each component of $\mu_{1}(\tau, 0)$ in the molecular frame about its mean value, so that the above average can be factored. Then in the low-field limit we have

$$
\left\langle\left[\mu_{1}(\tau, 0) \cdot \mathbf{e}_{i}\right]\left(\mathbf{e}_{\|} \cdot \mathbf{e}_{i}\right)\right\rangle_{E}=\left\langle\mu_{1}(\tau, 0) \cdot \mathbf{e}_{i}\right\rangle_{0}\left\langle\mathbf{e}_{\|} \cdot \mathbf{e}_{i}\right\rangle_{E}
$$

since $\left\langle\mathbf{e}_{\| \mid} \cdot \mathbf{e}_{i}\right\rangle_{0}=0$. But clearly $\left\langle\boldsymbol{\mu}_{\mathbf{t}}(\tau, 0) \cdot \mathbf{e}_{i}\right\rangle_{0}$ is zero unless $i=1$, so that (2) becomes finally

$$
[(\epsilon-1) /(\epsilon+2)](3 / 4 \pi \rho)=\mu_{e}\left[\left\langle\cos \theta_{1}\right\rangle_{E} / E\right]+\bar{\alpha}_{e},
$$

where

$$
\begin{gathered}
\mu_{e}=\left\langle\mu_{1} \cdot \mathbf{e}_{1}\right\rangle_{0}, \\
3 \vec{\alpha}_{e}=\left\langle\operatorname{Tr}\left[\partial \mu_{1}(\tau, \mathbf{E}) / \partial \mathbf{E}\right]_{E=0}\right\rangle_{0},
\end{gathered}
$$

and we have used the equivalence of the $x, y$, and $z$ laboratory axes in zero field to simplify the term $\bar{\alpha}_{e}$. The quantities $\mu_{e}$ and $\bar{\alpha}_{e}$ can be regarded as the effective permanent moment and mean molecular polarizability of molecule 1 . These quantities, particularly the effective moment $\mu_{e}$, can differ appreciably from their gasphase values since in a liquid molecule 1 is in strong interaction with its neighbors for almost all probable configurations. In large part, this strong interaction may be regarded as a reaction-field effect: The reaction field results from moments induced in the surrounding medium by the field of molecule 1 , and acts to polarize molecule 1 further even in the absence of the applied field. The qualitative nature of this effect is well represented in the Onsager model ${ }^{4}$ of a polar liquid.

The neglect of fluctuations in the development leading to Eq. (3) is essentially equivalent to approximations made by Kirkwood ${ }^{1}$ and by Harris and Alder ${ }^{5}$; these approximations have been discussed by Harris. ${ }^{6}$ We note from $\mathrm{Eq}$. (4a) that the effective permanent moment is simply the average projection of the total moment on the permanent moment direction. This definition was also introduced by Harris. ${ }^{6}$

We see from Eq. (3) that measurement of the dielectric constant allows one to determine the polarization provided that $\mu_{e}$ and $\bar{\alpha}_{e}$ are known or can be estimated. There are at least two useful ways of obtaining an approximate value for $\bar{\alpha}_{e}$ : (a) If we require that Eq. (3) reduce to the Clausius-Mossotti equation for nonpolar molecules (note that $\mu_{e}$ is zero if $\mu^{0}$ is zero), we are led to identify $\bar{\alpha}_{e}$ with $\bar{\alpha}$, the mean polarizability of the isolated molecule. This approxima. tion is equivalent to the use of the Lorentz local field ${ }^{7}$ $\mathbf{F}=(\epsilon+2) \mathbf{E}_{m} / 3$ in the defining Eq. (4b). (b) The most common way of dealing with $\bar{\alpha}_{e}$ is to introduce another macroscopic parameter, the high-frequency dielectric constant $\epsilon_{\omega}$. If we apply a sinusoidal electric field whose angular frequency $\omega$ is both high enough that $\left\langle\cos \theta_{1}\right\rangle_{E}$ is zero (i.e., the molecules do not have time to line up with the field) but low enough that $\bar{\alpha}_{e}$ differs negligibly from its zero-frequency value (assuming that it is possible to satisfy both conditions simultaneously), then

$$
\bar{\alpha}_{e}=\left[\left(\epsilon_{\omega}-1\right) /\left(\epsilon_{\omega}+2\right)\right](3 / 4 \pi \rho),
$$

where $\epsilon_{\omega}$ is the dielectric constant at angular frequency $\omega$. Equation (3) can then be written as

$$
\left(\frac{3}{\epsilon_{\omega}+2}\right)\left(\frac{\epsilon-\epsilon_{\omega}}{\epsilon+2}\right)\left(\frac{3}{4 \pi \rho}\right)=\mu_{e} \frac{\left\langle\cos \theta_{1}\right\rangle_{E}}{E} .
$$

As a good working approximation, we can identify $\epsilon_{\omega}$ with $n^{2}$, the square of the optical index of refraction. By doing this, we conclude that for typical highly polar liquids the $\bar{\alpha}_{e}$ term in (3) contributes roughly $25 \%$ of the total.

The estimation of $\mu_{e}$ is a much more difficult problem. If something is known about the local structure of the liquid, the reaction field can be estimated by the pro- 
cedure used by Kirkwood ${ }^{1}$ for water, and the induced moment calculated from this field and the relevant electrical parameters (e.g., polarizability, first hyperpolarizability, etc.) of the molecule. In the absence of other information, $\mu_{e}$ may be estimated using the Onsager model, ${ }^{4}$ for which

$$
\mu_{e}=\mu^{0}\left[\left(n^{2}+2\right)(2 \epsilon+1)\right] /\left[3\left(2 \epsilon+n^{2}\right)\right] .
$$

Equation (6) can be expected to provide only the crudest estimate of $\mu_{e}$, since the Onsager model takes no account of molecular shape, hyperpolarizability, or short-range order. Each of these effects would be expected to influence $\mu_{e}$ appreciably.

\section{THE NMR ELECTRIC-FIELD EFFECT}

The occurrence of the alignment rather than the polarization in NMR effects can be regarded as arising from the fact that magnetic dipole and electric quadrupole interactions of the nuclei, rather than electric dipole interactions, are involved. The spin Hamiltonians which appear in NMR can in general be written ${ }^{8}$

$$
H_{S}=\sum_{q} F_{q} A^{(q)},
$$

where $F_{q}$ is a function, expressible in spherical tensor form, of the orientation of the nuclear surroundings in the external magnetic field $\mathrm{H}_{0}$, and $A^{(q)}$ is a collection of spin angular momentum operators. The part of $H_{S}$ which must be kept to calculate the steady-state radiofrequency absorption spectrum of a fluid is obtained by averaging the appropriate $F_{q}$ over all molecular positions and orientations representative of the fluid. ${ }^{8}$ As a single example, we give $H_{S}$ for a nucleus of spin $I \geq 1$ whose quadrupole moment is coupled to an axially symmetric electric field gradient, such as might exist if the nucleus resides in a single covalent chemical bond:

$H_{S}=\left[e^{2} q Q / 4 I(2 I-1)\right]\left\langle P_{2}\left(\cos \psi_{1}\right)\right\rangle\left(\mathbf{I} \cdot \mathbf{I}-3 I_{z}^{2}\right)+Z$.

Here $e^{2} q Q$ is the quadrupole coupling constant, $\psi_{1}$ is the angle between $\mathbf{H}_{0}$ and the axis of symmetry of the molecular electric-field gradient, and $Z$ is the Zeeman energy, assumed large. Similar expressions can be written for many other observable nuclear interactions, including the direct magnetic dipole-dipole coupling, the anisotropic chemical shielding, etc.

In an ordinary fluid, many interactions, including that of Eq. (8), vanish because of the isotropy of the fluid;

$$
\left\langle P_{2}\left(\cos \psi_{1}\right)\right\rangle_{0}=0 \text {. }
$$

Here the subscript indicates that the average is to be taken in the unperturbed fluid. However, a fluid, especially a polar fluid, subject to an externally applied electric field $\mathbf{E}$ is slightly aligned. Applying the addition theorem for spherical harmonics ${ }^{2}$ and making use of the axial symmetry of the problem, we see that NMR spectra now contain information about the molecular alignment through

$$
\left\langle P_{2}\left(\cos \psi_{1}\right)\right\rangle_{E}=\left\langle P_{2}\left(\cos \theta_{1}\right)\right\rangle_{E} P_{2}(\cos \phi) P_{2}(\cos \delta),
$$

where $\phi$ is the angle between $\mathbf{E}$ and $\mathbf{H}_{0}$ and $\delta$ is the angle in the molecule between the permanent moment $\mu_{1}{ }^{0}$ and the field-gradient axis referred to above. The possibility of observing NMR effects of this kind has been suggested by a number of authors, ${ }^{9-13}$ and they have recently been convincingly demonstrated experimentally. ${ }^{14}$

Our interest in such experiments lies in the fact that, for molecules of known structure $(\delta)$ and properties (e.g., $e^{2} q Q$ ), they provide a measure of the molecular alignment $\left\langle P_{2}\left(\cos \theta_{1}\right)\right\rangle_{E}$ which is caused by an electric field. The significance of this information, when coupled with measurements of the dielectric constant, may be illustrated as follows ${ }^{15}$ : Consider a fluid in which the effects on each molecule of the applied field $\mathbf{E}$, the fields of surrounding dipoles, and all other intermolecular forces can be represented by the action of a single constant "effective" field $\mathbf{F}$ proportional to $\mathbf{E}_{m}$. This approximation is of course trivially correct for very dilute gases, where $\mathbf{F}=\mathbf{E}=\mathbf{E}_{m}$, but is frequently made for dense systems, ${ }^{16}$ where a more complicated relationship is said to exist between $\mathbf{E}_{m}$ and F. ${ }^{17}$ The angular distribution of the molecules is then governed by the Boltzmann factor

$$
\exp \left\{\beta\left[\boldsymbol{\mu}^{\mathbf{0}} \cdot \mathbf{F}+\frac{1}{2} \mathbf{F} \cdot \mathbf{a} \cdot \mathbf{F}+\mathcal{O}\left(F^{3}\right)\right]\right\},
$$

where $\boldsymbol{\mu}^{0}$ and $a$ are by hypothesis the ordinary gasphase permanent moment and polarizability, and $\beta \equiv 1 / k T$. Now for axially symmetric molecules, a standard rotational transformation ${ }^{18}$ yields

$$
\begin{gathered}
\boldsymbol{\mu}^{0} \cdot \mathbf{F}=\mu^{0} F \cos \theta_{1}, \\
\mathbf{F} \cdot \mathbf{a} \cdot \mathbf{F}=\left[\bar{\alpha}+(2 / 3)\left(\alpha_{||}-\alpha_{\perp}\right) P_{2}\left(\cos \theta_{1}\right)\right] F^{2},
\end{gathered}
$$

where $\bar{\alpha}=(1 / 3) \operatorname{Tr} a, \alpha_{\| 1}=a: e_{1} e_{1}$, and $\alpha_{\perp}=a: e_{2} e_{2}$. An elementary calculation then yields for the polarization and alignment

$$
\begin{gathered}
\left\langle\cos \theta_{1}\right\rangle_{E}=(1 / 3) \beta \mu^{0} F, \\
\left\langle P_{2}\left(\cos \theta_{1}\right)\right\rangle_{E}=(1 / 15) \beta F^{2}\left[\beta \mu^{02}+\left(\alpha_{\mid 1}-\alpha_{\perp}\right)\right],
\end{gathered}
$$

where the low-field limit has again been invoked. If only one of these quantities were measured experimentally, through the dielectric constant by means of (5) or through NMR effects by means of (9), it could be fitted into a consistent picture by suitable choice of the effective field $\mathbf{F}$. If both are measured, this freedom is lost. Define

$$
\eta=\left\langle P_{2}\left(\cos \theta_{1}\right)\right\rangle_{E} /\left\langle\cos \theta_{1}\right\rangle_{E}^{2} .
$$

Since $\left(\alpha_{||}-\alpha_{\perp}\right)$ is typically much less than $\beta \mu^{02}$, the constant effective field model predicts that $\eta$ be very close to $\mathbf{0 . 6 0}$, independent of the relation of $\mathbf{F}$ to $\mathbf{E}_{m}$. Moreover, if this model is valid, then $\eta$ should be independent of temperature, even if the local field $\mathbf{F}$ is 
temperature dependent. We have considered in this discussion only local fields $\mathbf{F}$ which are proportional to $\mathbf{E}_{m}$; if we also allowed for a reaction field, as Onsager did, the effect would be to replace $\mu^{0}$ and $\mathbf{a}$ by appropriate effective parameters (not necessarily the same as those previously defined), but this does not affect $\eta$ since the parameters cancel out.

To date positive results for the NMR electric field experiment have been obtained for the following liquids: $p$-nitrotoluene, nitrobenzene, nitromethane, deuteronitrobenzene, acetonitrile, propionitrile, isobutyronitrile, and chloroacetonitrile..$^{19,14}$ Dielectric data are also available for most of these compounds, but in order to estimate $\left\langle\cos \theta_{1}\right\rangle_{E}$ from the dielectric constant, we must first estimate $\mu_{e}$ [see Eq. (5)]. As previously mentioned, the Onsager model can be expected to provide only a crude estimate of the magnitude of $\mu_{e}$. Since $\eta$ involves $\left\langle\cos \theta_{1}\right\rangle_{E}$ squared, it is clearly necessary to consider the value of $\mu_{e}$ with some care if one wishes to obtain reliable $\eta$ values by combining experimental dielectric and NMR data. Since $\mu_{e}$ must be separately considered for each substance, any attempt to obtain reasonable $\mu_{e}$ values for the above substances would represent a long digression which would take us away from the central relationships which we wish to clarify. We therefore make no attempt here to extract reliable $\eta$ values from the experimental data. However, rather crude considerations suffice to indicate the inadequacy of the effective-field model. Define $\eta_{0}$ as the value of $\eta$ implied by the experimental dielectric and NMR data when the dielectric data are interpreted by replacing $\mu_{e}$ by $\mu^{0}$ and neglecting $\bar{\alpha}_{e}$ in Eq. (3). It is clear that $\eta_{0}$ is a poor approximation to $\eta$, since as previously mentioned $\bar{\alpha}_{e}$ is typically about $25 \%$ of the right-hand side of (3), and since the Onsager model indicates that $\mu_{e}$ is somewhere in the neighborhood of $1.3 \mu^{0}$ or $1.4 \mu^{0}$ in typical cases. Both of these effects tend to make $\eta$ greater than $\eta_{0}$, typically by a factor of two or three. Now $\eta_{0}$ can readily be calculated; one finds that for $p$-nitrotoluene at about $70^{\circ} \mathrm{C} \eta_{0}=4.2$, while for nitrobenzene, nitromethane, acetonitrile, propionitrile, and isobutyronitrile at room temperature the $\eta_{0}$ values cluster between 0.58 and 0.82 . Even in so crude a discussion, three points deserve mention: (a) The behavior of $p$-nitrotoluene is highly anomalous in comparison with the behavior of the other liquids. (b) The $\eta_{0}$ values for the other liquids are fortuitously near the value of $\eta$ appropriate to the effective-field model. (c) Since $\eta$ is probably at least $2 \eta_{0}$, none of the above compounds appears to be adequately described by the effective-field model. This would seem to indicate that short-range intermolecular forces are involved in an essential way, and that experimental values of both the polarization and alignment taken together provide a sensitive means of investigating them.

\section{THE KERR EFFECT}

The Kerr effect has sometimes been considered to be sensitive to the alignment in the same fashion as the
NMR experiment. In this section we will derive an expression for the Kerr constant and will show that the Kerr effect is sensitive to the polarization as well.

The molecular Kerr constant $K$ is customarily defined ${ }^{20}$ by

$$
K=6 n /\left[\rho\left(n^{2}+2\right)^{2}(\epsilon+2)^{2}\right] \lim _{\mathbf{E}_{m \rightarrow 0}}\left[\left(n_{\mid !}-n_{\perp}\right) / E_{m}^{2}\right],
$$

where $n_{\|}$and $n_{\perp}$ are the refractive indices for light polarized parallel and perpendicular to $\mathbf{E}_{m}$, respectively, and $n \equiv(1 / 2)\left(n_{\|}+n_{\perp}\right)$. The quantity $\left(n_{||}-n_{\perp}\right)$ is called the birefringence.

There are now two external electric fields in the problem, the static field $\mathbf{E}$ and the optical field $\mathbf{E}_{\omega}$ of the incident light wave. We therefore must write $\boldsymbol{\mu}_{1}$ as $\boldsymbol{\mu}_{1}\left(\tau, \mathbf{E}, \mathbf{E}_{\omega}\right)$; we cannot write $\mu_{1}\left(\tau, \mathbf{E}+E_{\omega}\right)$ because of the difference in frequency of the two electric fields. At optical frequencies the dielectric constant is the square of the refractive index and $\left\langle\cos \theta_{1}\right\rangle_{E}$ is zero, so that a trivial modification of $\mathrm{Eq}$. (3) yields

$$
\begin{aligned}
& {\left[\left(n_{p}^{2}-1\right) /\left(n_{p}^{2}+2\right)\right](3 / 4 \pi \rho)} \\
& =\left\langle\left[\partial \mu_{1}\left(\tau, \mathbf{E}, \mathbf{E}_{\omega}\right) / \partial \mathbf{E}_{\omega}\right]_{E_{\omega}=0}: \mathbf{e}_{p} \mathbf{e}_{p}\right\rangle_{E},
\end{aligned}
$$

where $\mathbf{e}_{p}$ is a unit vector in the direction of polarization of a plane-polarized light beam and $n_{p}$ is the refractive index in the presence of the static field for light of this polarization. Here $\mathbf{e}_{p}$ may be either $\mathbf{e}_{\|}$or $\mathbf{e}_{\perp}$, where $\mathbf{e}_{\perp}$ is a unit vector orthogonal to both $\mathbf{e}_{\|}$and the $\mathbf{k}$ vector of the incident light beam. Now from (12) and (13) we obtain

$$
K=(4 \pi / 27)\left\langle\left[\mathbf{a}_{\omega}(\tau, \mathbf{E}):\left(\mathbf{e}_{\|} \mathbf{e}_{\|}-\mathbf{e}_{\perp} \mathbf{e}_{\perp}\right)\right\rangle_{E} / E^{2}\right],
$$

where $a_{\omega}(\tau, \mathbf{E})$ is the high-frequency differential polarizability of molecule 1 in the presence of the other molecules and the field $\mathbf{E}$, defined by

$$
\mathbf{a}_{\omega}(\tau, \mathbf{E})=\left[\partial \mu_{1}\left(\tau, \mathbf{E}, \mathbf{E}_{\omega}\right) / \partial \mathbf{E}_{\omega}\right]_{E_{\omega}=0} .
$$

We now expand $\mathbf{a}_{\omega}(\tau, \mathbf{E})$ in powers of $\mathbf{E}$ to obtain

$$
\mathbf{a}_{\omega}(\tau, \mathbf{E})=\mathbf{a}_{\omega}(\tau)+\mathbf{E} \cdot \mathbf{B}_{\omega}(\tau)+\cdots,
$$

where

$$
\begin{gathered}
\mathbf{a}_{\omega}(\tau)=\mathbf{a}_{\omega}(\tau, 0), \\
\mathbf{B}_{\omega}(\tau)=\left[\partial^{2} \mu_{1}\left(\tau, \mathbf{E}, \mathbf{E}_{\omega}\right) / \partial \mathbf{E} \partial \mathbf{E}_{\omega}\right]_{E=E_{\omega}=0} .
\end{gathered}
$$

Unlike the ordinary first hyperpolarizability of Appen$\operatorname{dix} A, B_{\omega}(\tau)$ need not be symmetric, but we will assume for simplicity that it can be replaced by an equivalent symmetric tensor. From (14) and (16), we have

$K=(4 \pi / 27)\left[\left\langle\mathrm{a}_{\omega}(\tau): \mathbf{e}\right\rangle_{E} / E^{2}+\left\langle\mathbf{e}_{\| \mid} \cdot \mathrm{B}_{\omega}(\tau): \mathrm{e}\right\rangle_{E} / E\right]$,

where $\mathbf{e} \equiv \mathbf{e}_{\|} \mathbf{e}_{\|}-\mathbf{e}_{\perp} \mathbf{e}_{\perp}$. Now just as in Sec. II we expand the above in the molecular basis set $\left\{\mathbf{e}_{\mathbf{i}}\right\}$ and break the average, which means that we neglect fluctuations in the components of $a_{\omega}(\tau)$ and $B_{\omega}(\tau)$ in the molecular frame 
about their mean values. Then, we have

$$
\begin{aligned}
& \left\langle\mathbf{a}_{\omega}(\tau): \mathbf{e}\right\rangle_{E}=\sum_{i j}\left\langle\mathbf{a}_{\omega}(\tau): \mathbf{e}_{i} \mathbf{e}_{j}\right\rangle_{E}\left\langle\mathbf{e}: \mathbf{e}_{i} \mathbf{e}_{j}\right\rangle_{E}, \\
& \left\langle\mathbf{B}_{\omega}(\tau) \vdots \mathbf{e} \mathbf{e}_{\| \mid}\right\rangle_{E}=\sum_{i j k}\left\langle\mathbf{B}_{\omega}(\tau) \vdots \mathbf{e}_{i} \mathbf{e}_{j} \mathbf{e}_{k}\right\rangle_{E}\left\langle\left(\mathbf{e}_{\|} \cdot \mathbf{e}_{k}\right)\left(\mathbf{e}: \mathbf{e}_{i} \mathbf{e}_{j}\right)\right\rangle_{E} .
\end{aligned}
$$

The quantity $\left\langle\mathbf{e}: \mathbf{e}_{i} \mathbf{e}_{j}\right\rangle_{E}$ is of order $E^{2}$ in the low-field limit, since it vanishes for $\mathbf{E}=0$ and is invariant to reversal of the field. It is also clear that $\left\langle\left(\mathbf{e}_{\|} \cdot \mathbf{e}_{k}\right) \times\right.$ $\left.\left(\mathbf{e}: \mathbf{e}_{i} \mathbf{e}_{j}\right)\right\rangle_{0}$ is zero, so that in the low-field limit the above can be written

$$
\begin{gathered}
\left\langle\mathbf{a}_{\omega}(\tau): \mathbf{e}\right\rangle_{E}=\sum_{i j}\left\langle\mathbf{a}_{\omega}(\tau): \mathbf{e}_{i} \mathbf{e}_{j}\right\rangle_{0}\left\langle\mathbf{e}: \mathbf{e}_{i} \mathbf{e}_{j}\right\rangle_{E}, \\
\left\langle B_{\omega}(\tau) \vdots \mathbf{e} \mathbf{e}_{\| 1}\right\rangle_{E}=\sum_{i j k}\left\langle B_{\omega}(\tau): \mathbf{e}_{i} \mathbf{e}_{j} \mathbf{e}_{k}\right\rangle_{0}\left\langle\left(\mathbf{e}_{\|} \cdot \mathbf{e}_{k}\right)\left(\mathbf{e}: \mathbf{e}_{i} \mathbf{e}_{j}\right)\right\rangle_{E} .
\end{gathered}
$$

We now define effective optical-frequency polarizability and first hyperpolarizability tensors $\boldsymbol{a}_{\omega e}$ and $\mathbf{B}_{\omega e}$ by

$$
\begin{aligned}
& \mathbf{a}_{\omega e}=\sum_{i j} \mathbf{e}_{i} \mathbf{e}_{j}\left\langle\mathbf{a}_{\omega}(\tau): \mathbf{e}_{i} \mathbf{e}_{j}\right\rangle_{0}, \\
& \mathbf{B}_{\omega \boldsymbol{e}}=\sum_{i j k} \mathbf{e}_{i} \mathbf{e}_{j} \mathbf{e}_{k}\left\langle\mathbf{B}_{\omega}(\tau) \vdots \mathbf{e}_{i} \mathbf{e}_{j} \mathbf{e}_{k}\right\rangle_{\mathbf{0}} .
\end{aligned}
$$

Because of the axial symmetry, each of these tensors has only two independent components in the molecular frame, ${ }^{21}$. given by

$$
\begin{aligned}
& \alpha_{\omega e}{ }^{\prime \prime}=a_{\omega e}: \mathbf{e}_{1} \mathbf{e}_{1}, \\
& \alpha_{\omega e} \perp=a_{\omega e}: \mathbf{e}_{2} \mathbf{e}_{2}, \\
& \beta_{\omega e}{ }^{\prime \prime}=B_{\omega e}: e_{1} e_{1} e_{1}, \\
& \beta_{\omega e}{ }^{\perp}=B_{\omega e}: e_{1} e_{2} e_{2} .
\end{aligned}
$$

We now have

$$
\begin{aligned}
\left\langle a_{\omega}(\tau): e\right\rangle_{E} & =\left\langle a_{\omega e}: e\right\rangle_{E}, \\
\left\langle B_{\omega}(\tau) \vdots e e_{\| 1}\right\rangle_{E} & =\left\langle B_{\omega e}: e e_{\| 1}\right\rangle_{E},
\end{aligned}
$$

and a standard rotational transformation ${ }^{18}$ yields

$$
\begin{aligned}
\left\langle\mathbf{a}_{\omega e}: \mathrm{e}\right\rangle_{E} & =\Delta \alpha_{\omega e}\left\langle P_{2}\left(\cos \theta_{1}\right)\right\rangle_{E}, \\
\left\langle\mathbf{B}_{\omega e}: \mathbf{e} \mathbf{e}_{\| 1}\right\rangle_{E} & =(2 / 3) \bar{\beta}_{\omega e}\left\langle\cos \theta_{1}\right\rangle_{E},
\end{aligned}
$$

where

$$
\begin{aligned}
& \Delta \alpha_{\omega e}=\left(\alpha_{\omega e}{ }^{\prime \prime}-\alpha_{\omega e} \mathcal{L}\right), \\
& \bar{\beta}_{\omega e}=(3 / 5)\left(\beta_{\omega e} \|+2 \beta_{\omega \epsilon} \mathcal{L}\right) \text {. }
\end{aligned}
$$

We have neglected in (20b) a term proportional to $\left\langle P_{3}\left(\cos \theta_{1}\right)\right\rangle_{E}$ since it is of order $E^{3}$ and is therefore negligible in the low-field limit. Our final equation for the Kerr constant now becomes

$$
\begin{aligned}
K=(4 \pi / 27)\left\{\Delta \alpha_{\omega e}\left[\left\langle P_{2}\left(\cos \theta_{1}\right)\right\rangle_{E} / E^{2}\right]\right. \\
\left.+\frac{2}{3} \bar{\beta}_{\omega e}\left(\left\langle\cos \theta_{1}\right\rangle_{E} / E\right)\right\} .
\end{aligned}
$$

Equation (22) shows that the existence of molecular hyperpolarizability destroys the equivalence of the Kerr and NMR experiments. It is qualitatively apparent that the hyperpolarizability term may be significant since it comes into the Kerr effect through the polarization $\left\langle\cos \theta_{1}\right\rangle_{E}$, which is linear in the field.

Once again we have the problem of how to approximate the effective molecular parameters. We first consider $\Delta \alpha_{w e}$. Again, two possible approaches suggest themselves: (a) By evaluating (13) for the case $\mathbf{E}=\mathbf{0}$, we obtain the following expression for the ordinary refractive index:

$$
\begin{aligned}
{\left[\left(n^{2}-1\right) /\left(n^{2}+2\right)\right](3 / 4 \pi \rho)=\frac{1}{3}\left\langle\operatorname{Tr}\left(\partial \mu_{1} / \partial \mathrm{E}_{\omega}\right)_{E_{\omega}=0}\right\rangle_{0} } & \\
=\frac{1}{3} \operatorname{Tra}_{\omega e} & \equiv \bar{\alpha}_{\omega \theta} .
\end{aligned}
$$

If we require this to be consistent with the LorenzLorentz equation, we are led to identify $\bar{\alpha}_{\omega e}$ with $\bar{\alpha}_{\omega}$, the mean optical polarizability of the isolated molecule. It is therefore not unreasonable to equate $\Delta \alpha_{\omega e}$ with $\Delta \alpha_{\omega}$, the polarizability anisotropy for the isolated molecule. (b) A better approach is to introduce the refractive index as a macroscopic parameter. It is necessary to assume that the relative anisotropy is the same for both the effective and gas-phase optical polarizabilities:

$$
\Delta \alpha_{\omega e} / \bar{\alpha}_{\omega e}=\Delta \alpha_{\omega} / \bar{\alpha}_{\omega} .
$$

This assumption was also implicit in (a) above. Then we have

$$
\Delta \alpha_{\omega e}=\left(\frac{\Delta \alpha_{\omega}}{\bar{\alpha}_{\omega}}\right)\left(\frac{n^{2}-1}{n^{2}+2}\right)\left(\frac{3}{4 \pi \rho}\right) .
$$

For axially symmetric molecules, $\left(\Delta \alpha_{\omega} / \bar{\alpha}_{\omega}\right)$ can be obtained directly by measuring the depolarization ratio $\Delta$ for light scattered from the ideal gas $^{22}$ : $\left(\Delta \alpha_{\omega} / \bar{\alpha}_{\omega}\right)=[45 \Delta /(6-7 \Delta)]^{1 / 2}$.

There is unfortunately no very satisfactory method of approximating $\bar{\beta}_{\omega e}$. If we were to assume that

$$
\left\langle\left(\partial^{2} \mu_{1} / \partial \mathbf{E} \partial \mathbf{E}_{\omega}\right)_{0}: \mathbf{e}_{i} \mathbf{e}_{j} \mathbf{e}_{k}\right\rangle_{0} \cong\left\langle\left(\partial^{2} \mu_{1} / \partial \mathbf{E}_{\omega} \partial \mathbf{E}_{\omega}\right)_{0}: \mathbf{e}_{i} \mathbf{e}_{j} \mathbf{e}_{k}\right\rangle_{0},
$$

then we could relate $\bar{\beta}_{\omega e}$ to the quadratic coefficient of optical-frequency dielectric saturation; but, since this quantity is not well known there seems little advantage in doing this. We may obtain a very rough estimate of $\bar{\beta}_{\omega e}$ by using the Onsager cavity field ${ }^{4} 3 \epsilon \mathrm{E}_{m} /(2 \epsilon+1)$ for the static local field seen by molecule 1 and the Lorentz local field ${ }^{7}\left(n^{2}+2\right) \mathbf{E}_{m \omega} / 3$ for the optical local field seen by molecule 1 in the defining Eq. (17b). The result of doing this is

$$
\bar{\beta}_{\omega e} \cong \bar{\beta}_{\omega}[9 \epsilon /(2 \epsilon+1)(\epsilon+2)],
$$

where $\bar{\beta}_{\omega}$ is the gas-phase value of the mean optical molecular first hyperpolarizability. Because of the local-field approximations made in its derivation, Eq. (24) cannot be trusted to give more than a crude estimate of the magnitude of $\bar{\beta}_{\omega e}$; it is somewhat analogous to Eq. (6).

Combining (22) with (5) and making the approximation (23), we see that the dielectric constant, the Kerr constant, and the NMR experiment are interrelated according to (25):

$$
\begin{array}{r}
K=\left[(\epsilon+2)\left(n^{2}+2\right) \rho\right]^{-1}\left[\left(\frac{n^{2}-1}{\epsilon+2}\right)\left(\frac{\Delta \alpha_{\omega}}{\bar{\alpha}_{\omega}}\right) \frac{\left\langle P_{2}\left(\cos \theta_{1}\right)\right\rangle_{E}}{E_{m}^{2}}\right. \\
\left.+\frac{2}{9}\left(\frac{\bar{\beta}_{\omega \epsilon}}{\mu_{e}}\right)\left(\epsilon-n^{2}\right)\right],
\end{array}
$$


TABLE I. Apparent discrepancies between Kerr and NMR data.

\begin{tabular}{|c|c|c|c|c|c|c|c|c|c|}
\hline Substance & $\epsilon$ & $n$ & $\boldsymbol{\mu}^{0}(D)$ & $\begin{array}{c}\alpha_{\omega} \times 10^{24} \\
(\mathrm{esu})\end{array}$ & $\begin{array}{c}\Delta \alpha_{\omega} \times 10^{24} \\
(\mathrm{esu})^{\mathrm{b}}\end{array}$ & $\begin{array}{c}\left\langle P_{2}\right\rangle_{E} / E_{m}{ }^{2} \\
\times 10^{8}(\mathrm{esu}) \\
\text { from NMR } \\
\quad \text { data }\end{array}$ & $\begin{array}{c}\left\langle P_{2}\right\rangle_{E} / E_{m}{ }^{2} \\
\times 10^{8}(\mathrm{esu}) \\
\text { from Kerr } \\
\text { dataj using } \\
(25) \text { with } \\
\bar{\beta}_{\omega e}=0\end{array}$ & $\begin{array}{c}\bar{\beta}_{\omega e} / \mu_{e} \\
\times 10^{12}(\mathrm{esu})\end{array}$ & $\begin{array}{c}\text { Very } \\
\text { approximate } \\
\bar{\beta}_{\omega} \times 10^{2 g} \\
(\mathrm{esu})^{\mathrm{c}}\end{array}$ \\
\hline Nitromethane & $37^{\mathrm{d}}$ & $1.38^{\mathrm{i}}$ & $3.46^{\circ}$ & 4.8 & $0.8^{8}$ & $0.36^{\mathrm{i}}$ & 0.088 & -1.33 & -5.2 \\
\hline Nitrobenzene & $35^{\mathrm{e}}$ & $1.56^{\mathrm{f}}$ & $4.22^{\mathrm{c}}$ & $12.9^{\mathrm{b}}$ & $7.3^{\mathrm{b}}$ & $0.97^{\mathrm{i}}$ & 0.61 & -11.0 & -56 \\
\hline
\end{tabular}

all data at room temperature.

b Approximated by $\alpha_{11}-\left(\alpha_{22}+\alpha_{33}\right) / 2$.

- Obtained by using (6) and (24).

d Handbook of Chemisiry, edited by N. A. Lange (McGraw-Hill, New York, 1961), 10th ed.

e Handbook of Chemistry and Physics, edited by R. W. Weast (Chemical Rubber, Cleveland, Ohio, 1969), 50th ed.

where we have identified $\epsilon_{\omega}$ with $n^{2}$ and have converted from $E$ back to $E_{m}$. In the next section, we use (25) to explain the apparent disagreement between some experimental Kerr and NMR alignment values.

\section{COMPARISON OF EXPERIMENTAL KERR AND NMR RESULTS}

Recently Hilbers and MacLean ${ }^{14}$ have successfully performed the NMR electric-field experiment and have used the results to infer reliable alignment values for a number of liquids, including nitrobenzene and nitromethane. Since the Kerr constants of these substances are also known, ${ }^{23}$ it becomes possible to compare alignment values inferred from two different sources. In the past, Kerr constants have often been interpreted under the assumption that $\bar{\beta}_{\omega e}$ is zero, partly because of the lack of reliable information about its size. This is what Hilbers and MacLean have done, and they find the Kerr alignment values thus obtained to differ from their NMR values. If we attribute the disagreement to the existence of a nonzero molecular hyperpolarizability, we can readily solve $(25)$ for the value of $\left(\bar{\beta}_{\omega e} / \mu_{e}\right)$ which resolves the discrepancy. In Table I we summarize the apparent discrepancies and the required values of $\left(\bar{\beta}_{\omega e} / \mu_{e}\right)$. We have also obtained very approximate values of $\bar{\beta}_{\omega}$ by making use of the crude approximations (6) and (24) to relate the effective permanent moment and hyperpolarizability to their gas-phase values. The values of $\bar{\beta}_{\omega}$ obtained in this way are certainly of the expected order of magnitude; except for Kielich's deduction ${ }^{24}$ of $\bar{\beta}=2 \times 10^{-28} \mathrm{esu}$ for $\mathrm{CHCl}_{3}$, both experimental ${ }^{25}$ and theoretical ${ }^{25,26}$ values of $\bar{\beta}$ for small molecules are of the order of $10^{-29}$ to $10^{-30} \mathrm{esu}$. The larger value in the case of nitrobenzene is not unexpected in view of the greater size and complexity of the molecule. We note that in the case of nitromethane a small value of $\left(\bar{\beta}_{\omega e} / \mu_{e}\right)$ suffices to explain an apparent disagreement of a factor of 4 . This is because the very small value of $\Delta \alpha_{\omega}$ for this substance causes the $\bar{\beta}_{\omega e}$ term to be relatively important by default.

\author{
f A. L. McClellan, Tables of Experimental Dipole Moments (Freeman, \\ San Francisco, 1963). \\ 8 Reference 27. \\ ${ }^{\text {b }}$ Reference 28. \\ i Reference 14. \\ i Reference 23.
}

The values of $\left(\bar{\beta}_{\omega e} / \mu_{e}\right)$ given in Table I should not, however, be taken too seriously, since we have, for simplicity, treated nitrobenzene and nitromethane as axially symmetric molecules, and since we have used the approximation (23). The former restriction could readily be alleviated, but there are more serious practical difficulties. Published values of the principal polarizabilities are customarily taken from experimental Kerr constants and depolarization ratios interpreted under the assumption that $\bar{\beta}_{\omega}=0 .{ }^{27,28}$ It is already apparent that $\bar{\beta}$ may make an important contribution to the Kerr effect, and Buckingham and Stephen ${ }^{29}$ have shown that it also contributes significantly to the depolarization ratios of polar substances. Thus presently available principal polarizabilities must be regarded with a certain amount of suspicion, and until these parameters become more reliably known, more complete interpretation of Kerr data will not be possible. Fortunately, measurement of the temperature dependence of the Kerr constant of a dilute polar gas can in principle be used to separate the contribution of the molecular polarizability from that of the hyperpolarizabilities, ${ }^{21}$ and other methods are also available for the experimental study of $\bar{\beta} .^{25}$ Of course, even if the gas-phase parameters are well known, we still have the problem that the true relation between them and the corresponding effective parameters is not known.

\section{STATISTICAL AVERAGES IN THE UNPERTURBED FLUID}

In order to investigate the influence on either the polarization or the alignment of intermolecular forces and short-range correlations, it is now necessary to reduce the various averages in the presence of the field, denoted by $\langle\cdots\rangle_{E}$, to zero-field averages $\langle\cdots\rangle_{0}$. Before doing this, however, we emphasize two important points: (a) We are considering a sphere of dielectric fluid of macroscopic size suspended in vacuum in a uniform applied dielectric field $\mathbf{E}$. The spherical sample is chosen for reasons of mathematical con- 
venience, and does not correspond to the usual experimental geometry for dielectric, Kerr, and NMR experiments, in which the dielectric fluid fills the region between the plates of a parallel-plate capacitor. However, we assume that the field $\mathbf{E}_{m}$ describes completely the macroscopic state of the dielectric, so that the dielectric constant, the Kerr constant, $\left\langle P_{2}\left(\cos \theta_{1}\right)\right\rangle_{E} / E_{m}{ }^{2}$, and $\left\langle\cos \theta_{1}\right\rangle_{E} / E_{m}$ are properties of the sample material, independent of sample shape. ${ }^{30} \mathrm{We}$ are therefore free to use any convenient geometry for their calculation. (b) Although we will deal only with the polarization and alignment in what follows, we emphasize that the effective parameters $\mu_{e}, a_{\omega e}$, and $\mathbf{B}_{\omega e}$ are also defined as statistical averages in zero field, and they also depend upon short-range correlations and intermolecular forces.

The definition of the average value of any dynamical variable $G(\tau, \mathbf{E})$ is given by

$$
\langle G(\tau, \mathbf{E})\rangle_{\boldsymbol{E}}=\frac{\int d \tau G(\tau, \mathbf{E}) \exp [-\beta U(\tau, \mathbf{E})]}{\int d \tau \exp [-\boldsymbol{\beta} U(\tau, \mathbf{E})]},
$$

where $U(\boldsymbol{\tau}, \mathbf{E})$ is the total potential energy of the sample in the configuration $\tau$ and in the presence of $\mathbf{E}$. It can be shown in general that

$$
U(\tau, \mathbf{E})=U_{0}(\tau)-\int_{0}^{\mathrm{E}} \mathbf{M}\left(\tau, \mathbf{E}^{\prime}\right) \cdot d \mathbf{E}^{\prime},
$$

where $U_{0}(\tau)$ is the potential energy of the configuration $\tau$ in zero field. This expression is quite generally valid for any molecular model, and automatically includes changes in dipole-dipole interaction energy induced by the field and intramolecular energy associated with field-induced molecular deformation. Since $\mathbf{E}$ is assumed uniform, only the dipole term appears in (27).

We now expand $\mathbf{M}$ in powers of $\mathbf{E}$, so that (27) becomes

$$
\begin{aligned}
U(\tau, \mathbf{E})=U_{0}(\tau) & -\mathbf{M}(\tau, 0) \cdot \mathbf{E} \\
& -\frac{1}{2} \mathbf{E} \mathbf{E}: \partial \mathbf{M}(\tau, \mathbf{E}) /\left.\partial \mathbf{E}\right|_{E=0}+\cdots .
\end{aligned}
$$

For rigid dipolar molecules, the expansion (28) ends with the linear term. For molecules with $a \neq 0$ but with B and higher-order hyperpolarizabilities equal to zero, the expansion ends with the quadratic term, and so on.

Using (26) and (28) and expanding $\langle\cdots\rangle_{E}$ in powers of $E$, it is easy to show that, in the low-field limit,

$$
\begin{aligned}
\left\langle\cos \theta_{1}\right\rangle_{E}= & (\beta E)\left\langle\left(\mathbf{e}_{1} \cdot \mathbf{e}_{\| \mid}\right)\left(\mathbf{M} \cdot \mathbf{e}_{\| \mid}\right)\right\rangle_{0}, \\
\left\langle P_{2}\left(\cos \theta_{1}\right)\right\rangle_{E}=\frac{1}{2} \beta E^{2} & {\left[\left\langle P_{2}\left(\mathbf{e}_{1} \cdot \mathbf{e}_{\| \mid}\right)(\partial \mathbf{M} / \partial \mathbf{E}): \mathbf{e}_{\| \mid} \mathbf{e}_{\| \mid}\right\rangle_{0}\right.} \\
& \left.+\beta\left\langle P_{2}\left(\mathbf{e}_{1} \cdot \mathbf{e}_{\| \mid}\right)\left(\mathbf{M} \cdot \mathbf{e}_{\| 1}\right)^{2}\right\rangle_{0}\right] .
\end{aligned}
$$

We have used the fact that zero-field averages $\langle\cdots\rangle_{0}$ must be invariant to the substitution $\mathbf{e}_{\|} \rightarrow-\mathbf{e}_{\|}$to eliminate terms which are identically zero from (29) and (30).

Since the sample is spherical, there is no preferred direction in the absence of the field. All the zero-field averages $\langle\cdots\rangle_{0}$ are therefore independent of the direction of $\mathbf{e}_{||}$and are unchanged by averaging the quantity within the brackets over this direction. Performing this "spherical average" on Eqs. (29) and (30) yields

$$
\begin{gathered}
\left\langle\cos \theta_{1}\right\rangle_{E} / E_{m}=\frac{1}{3}[(\epsilon+2) / 3] \beta\left\langle\mathbf{e}_{1} \cdot \mathbf{M}\right\rangle_{0}, \\
\left\langle P_{2}\left(\cos \theta_{1}\right)\right\rangle_{E} / E_{m}{ }^{2}=\frac{1}{30}[(\epsilon+2) / 3]^{2} \beta\left\{\beta \left[3\left\langle\left(\mathbf{e}_{1} \cdot \mathbf{M}\right)^{2}\right\rangle_{0}\right.\right. \\
\left.\left.-\langle\mathbf{M} \cdot \mathbf{M}\rangle_{0}\right]+3\left\langle\partial \mathbf{M} / \partial \mathbf{E}: \mathbf{e}_{1} \mathbf{e}_{\mathbf{1}}\right\rangle_{0}-\langle\operatorname{Tr} \partial \mathbf{M} / \partial \mathbf{E}\rangle_{0}\right\},
\end{gathered}
$$

where we have converted from $E$ to $E_{m}$ so that the left-hand sides of the above equations can be considered independent of sample geometry.

To proceed further we must adopt a molecular model, so that $\mathbf{M}(\tau, \mathbf{E})$ can be explicitly written down and dealt with, or adopt further simplifying assumptions. In the spirit of our previous approximations, we will neglect fluctuations in each molecular dipole moment about its average value in the quantities being averaged in (31) and (32). That is, we replace $\mathbf{M}$ by the sum of effective permanent molecular dipole moments. Then the last two terms in (32) vanish and the equations can be rewritten in a form which clearly displays the angular averages involved:

$$
\left\langle\cos \theta_{1}\right\rangle_{E} / E_{m}=\frac{1}{3}[(\epsilon+2) / 3] \beta \mu_{e}\left[1+(N-1)\left\langle\cos \gamma_{12}\right\rangle_{0}\right] \text {, }
$$

$$
\begin{gathered}
{\left[\left\langle P_{2}\left(\cos \theta_{1}\right)\right\rangle_{E}\right] / E_{m}^{2}=\frac{1}{15}[(\epsilon+2) / 3]^{2}\left(\beta \mu_{e}\right)^{2}} \\
\times\left[1+(N-1)\left\langle P_{2}\left(\cos \gamma_{12}\right)\right\rangle_{0}\right. \\
+\frac{3}{2}(N-1)(N-2)\left\langle\cos \gamma_{12} \cos \gamma_{13}\right\rangle_{0} \\
\left.-\frac{1}{2}(N-1)(N-6)\left\langle\cos \gamma_{12}\right\rangle_{0}\right]
\end{gathered}
$$

where $\gamma_{i j}$ is the angle between the principal axes (permanent moments) of molecules $i$ and $j$.

The left-hand sides of (33) and (34) are supposed independent of sample geometry. However, we have evaluated them for the particular choice of a spherical sample in vacuum, and this fact is reflected in their right-hand sides. In particular, we emphasize that for these expressions to be valid the zero-field averages $\langle\cdots\rangle_{0}$ must be performed over a spherical sample in vacuum. If we could rigorously perform such averages, our problem would be solved and the results (if our assumptions are valid) would be equally applicable to all experimental geometries. It is apparent that the evaluation of the averages in (33) and (34) would require knowledge of the two- and three-particle angledependent distribution functions..$^{31}$ Rigorous evaluation of these distribution functions is not feasible for dense systems. Moreover, the approximation and interpretation of expressions containing them is complicated by the fact that they appear to contain, in addition to the short-range correlations between a molecule and its nearest neighbors, long-range correlations (i.e., correlations between molecules separated by macroscopic distances) due to shape-dependent boundary effects (see Sec. VII). In our judgment, the macroscopic 
shape-dependent effects on fluid distribution functions due to long-range dipolar forces is a subject that has not been sufficiently investigated.

In order to arrive at expressions which are more easily approximated and which can be interpreted entirely on the basis of local-order considerations, it is desirable to deal with averages and distribution functions from which boundary effects are absent. To this end, we discuss in the next section the transformation of averages over a spherical sample in vacuum into averages devoid of boundary effects. We emphasize that this transformation is desirable purely from the standpoint of obtaining alternative expressions which can be more easily approximated and interpreted than (33) and (34). The expressions (33) and (34) are, within the framework of our assumptions, rigorous as they stand, and do not, for example, require modification in order to make the boundary effects which they contain correspond more closely to those present in the actual experimental geometry.

\section{BOUNDARY EFFECTS}

We must now deal with a problem which has led to a great deal of confusion in studies of dielectrics, not excepting the present one. We refer to the dependence of averages in a molecular theory upon the shape of the region over which they are performed, a dependence which arises basically from the long-range nature of the dipolar field. The existence of shape-dependence implies that the averages cannot be computed on the basis of local-order considerations alone. Some means must be found to separate out the shape-dependence if our goal of obtaining information about the purely local microscopic structure of the fluid is to be realized. Since sample shape is a macroscopic property of the system, it should not greatly surprise us if such a separation can be effected by suitable macroscopic considerations.

Kirkwood $^{1}$ has dealt with just this problem. He developed a procedure for transforming the average $\left\langle\mathbf{e}_{1} \cdot \mathbf{M}\right\rangle_{0}$ over a sphere in vacuum in zero field into an average $\left\langle\mathbf{e}_{\mathbf{1}} \cdot \mathbf{M}\right\rangle_{\infty}$ over a sphere in zero field immersed in an infinite medium of the same sample material. The boundary effects must be absent from $\langle\cdots\rangle_{\infty}$ since the boundary effectively disappears, and it is assumed proper to compute such averages entirely on a local basis. Kirkwood's analysis yields

$$
\left\langle\mathbf{e}_{1} \cdot \mathbf{M}\right\rangle_{0}=[3 /(\epsilon+2)][3 \epsilon /(2 \epsilon+1)]\left\langle\mathbf{e}_{1} \cdot \mathbf{M}\right\rangle_{\infty} .
$$

We note that elimination of the boundary effects is associated with multiplication by a factor depending only upon the dielectric constant, a macroscopic parameter, and not upon other molecular averages. Using (35) in (31) yields, instead of (33),

$$
\begin{aligned}
{\left[\left\langle\cos \theta_{1}\right\rangle_{E} / E_{m}\right]=\frac{1}{3} \beta \mu_{e}[} & 3 \epsilon /(2 \epsilon+1)] \\
\times & \times\left[1+(N-1)\left\langle\cos \gamma_{12}\right\rangle_{\infty}\right] .
\end{aligned}
$$

Kirkwood used (36) and (3) and a local-order estima- tion of $\left\langle\cos \gamma_{12}\right\rangle_{\infty}$ to estimate the dielectric constant of water. It is important to note that $3 \epsilon \mathrm{E}_{m} /(2 \epsilon+1)$ is the Onsager cavity field. In fact, it can easily be shown that if the short-range angular correlation term $\left\langle\cos \gamma_{12}\right\rangle_{\infty}$ is neglected in (36) and the result combined with (3) using the Onsager values ${ }^{7}$ for $\mu_{e}$ and $\bar{\alpha}_{e}$, the Onsager equation is obtained.

In order to deal with $\left\langle P_{2}\right\rangle_{E}$, given by (32), we need the transformation corresponding to (35) for the average $\left\langle\left(\mathbf{e}_{\mathbf{1}} \cdot \mathbf{M}\right)^{2}\right\rangle_{0}$. It turns out, however, that it is more convenient to deal with Eq. (30), the first term of which is now zero since we are replacing molecular dipole moments by effective permanent moments, and then perform the spherical average after the transformation $\langle\cdots\rangle_{0} \rightarrow\langle\cdots\rangle_{\infty}$ has been made. In Appendix $B$, we present a method for effecting the transformation from $\left\langle P_{2}\left(\mathbf{e}_{1} \cdot \mathbf{e}_{||}\right)\left(\mathbf{M} \cdot \mathbf{e}_{||}\right)^{2}\right\rangle_{0}$ to $\left\langle P_{2}\left(\mathbf{e}_{1} \cdot \mathbf{e}_{||}\right)\left(\mathbf{M} \cdot \mathbf{e}_{\| \mid}\right)^{2}\right\rangle_{\infty}$. In the process we rederive (35) in a way which we hope clarifies the legitimacy of Kirkwood's use of macroscopic electrostatics. The result is

$$
\begin{aligned}
\left\langle P_{2}\left(\mathbf{e}_{1} \cdot \mathbf{e}_{\| \mid}\right)\left(\mathbf{M} \cdot \mathbf{e}_{\|}\right)^{2}\right\rangle_{0}=\left[9_{\epsilon} /(2 \epsilon+1)(\epsilon+2)\right]^{2} & \\
& \times\left\langle P_{2}\left(\mathbf{e}_{1} \cdot \mathbf{e}_{\|}\right)\left(\mathbf{M} \cdot \mathbf{e}_{\| \mid}\right)^{2}\right\rangle_{\infty},
\end{aligned}
$$

so that (30) becomes

$$
\begin{aligned}
\left\langle P_{2}\left(\cos \theta_{1}\right)\right\rangle_{E} / E_{m}{ }^{2}=\frac{1}{2} \beta^{2}[3 \epsilon /(2 \epsilon & +1)]^{2} \\
& \times\left\langle P_{2}\left(\mathbf{e}_{1} \cdot \mathbf{e}_{\|}\right)\left(\mathbf{M} \cdot \mathbf{e}_{\|}\right)^{2}\right\rangle_{\infty} .
\end{aligned}
$$

We now perform the spherical average to obtain

$$
\begin{aligned}
\left\langle P_{2}\left(\cos \theta_{1}\right)\right\rangle_{E} / E_{m}{ }^{2}= & (1 / 30) \beta^{2}[3 \epsilon /(2 \epsilon+1)]^{2} \\
& \times\left[3\left\langle\left(\mathbf{e}_{1} \cdot \mathbf{M}\right)^{2}\right\rangle_{\infty}-\langle\mathbf{M} \cdot \mathbf{M}\rangle_{\infty}\right] .
\end{aligned}
$$

In terms of short-range angular correlations, (37) becomes, instead of (34),

$$
\begin{aligned}
& \left\langle P_{2}\left(\cos \theta_{1}\right)\right\rangle_{E} / E_{m}{ }^{2}=(1 / 15)\left(\beta \mu_{e}\right)^{2}[3 \epsilon /(2 \epsilon+1)]^{2} \\
& \quad \times\left[1+(N-1)\left\langle P_{2}\left(\cos \gamma_{12}\right)\right\rangle_{\infty}+\frac{3}{2}(N-1)(N-2)\right. \\
& \left.\times\left\langle\cos \gamma_{12} \cos \gamma_{13}\right\rangle_{\infty}-\frac{1}{2}(N-1)(N-6)\left\langle\cos \gamma_{12}\right\rangle_{\infty}\right] .
\end{aligned}
$$

Equations (36) and (38) reveal explicitly the effect of short-range angular correlations on the polarization and alignment. ${ }^{32}$

Expressions for the dielectric and Kerr constants in terms of short-range angular correlations in the unperturbed fluid are readily obtained by substituting (36) and (38) into (5) and (22).

We re-emphasize that the distribution functions needed for the calculation of the averages $\langle\cdots\rangle_{\infty}$ are those in which only local correlations are present. From (33) and (36) we note that $\left\langle\cos \gamma_{12}\right\rangle_{0} \neq\left\langle\cos \gamma_{12}\right\rangle_{\infty}$. Since the quantities being averaged are the same, the implication is that the difference must be due to a difference in the two-particle distribution functions appropriate to the two physical situations.

\section{APPROXIMATE EVALUATION OF AVERAGES}

The calculation from first principles of the two- and three-particle angular distribution functions (either 
ordinary or local) for liquids is an extremely complicated task and to our knowledge has not yet been attempted. This circumstance forces us to adopt further approximate procedures.

\section{A. Zero Correlation Approximation}

The simplest approximation which one might make is to assume that there is no short-range correlation whatever between the orientations of neighboring molecules. Then (36) becomes

$$
\left\langle\cos \theta_{1}\right\rangle_{E} / E_{m}=\frac{1}{3} \mu_{e} \beta[3 \epsilon /(2 \epsilon+1)],
$$

and, as previously mentioned, this leads to the Onsager result for the dielectric constant. In a similar manner (38) becomes

$$
\left\langle P_{2}\left(\cos \theta_{1}\right)\right\rangle_{E} / E_{m}^{2}=\frac{1}{15}\left(\beta \mu_{e}\right)^{2}[3 \epsilon /(2 \epsilon+1)]^{2} .
$$

Note that Eqs. (39) and (40) would have been obtained from Eqs. (10) by replacing $\mu^{0}$ by $\mu_{e}$ and by choosing $F$ to be the Onsager cavity field $3 \epsilon E_{m} /(2 \epsilon+1)$ [the polarizability term in (10b) does not appear because we have replaced molecular dipole moments by effective permanent moments].

It is of interest to obtain an approximate expression for the Kerr constant which is in some sense analogous to the Onsager expression ${ }^{4}$ for the dielectric constant. To do this we do the following: (a) We substitute (39) and (40) into (22). This corresponds to neglecting short-range angular correlations. (b) We use the Lorenz-Lorentz formula to relate $\bar{\alpha}_{\omega}$ to $n$ :

$$
\left[\left(n^{2}-1\right) /\left(n^{2}+2\right)\right](3 / 4 \pi \rho)=\bar{\alpha}_{\omega} .
$$

(c) We use the approximate expressions (6) and (24). The result of all this is

$$
\begin{aligned}
K=\frac{4 \pi}{243} & {\left[\frac{9 \epsilon}{(2 \epsilon+1)(\epsilon+2)}\right]^{2}\left(\frac{n^{2}+2}{3}\right)\left(\frac{2 \epsilon+1}{2 \epsilon+n^{2}}\right) } \\
\times & {\left[\frac{3}{5}\left(\frac{n^{2}+2}{3}\right)\left(\frac{2 \epsilon+1}{2 \epsilon+n^{2}}\right) \beta^{2} \mu^{02} \Delta \alpha_{\omega}+2 \beta \bar{\beta}_{\omega} \mu^{0}\right] . }
\end{aligned}
$$

It is of interest to compare Eq. (41) with the corresponding approximate expression of Buckingham and Raab $^{20}$ obtained from their equations (34), (35), and (41) :

$$
\begin{aligned}
& K_{B R}=\frac{4 \pi}{243}[\left.\frac{9 \epsilon}{(2 \epsilon+1)(\epsilon+2)}\right]^{2}\left(\frac{n^{2}+2}{3}\right)\left(\frac{2 \epsilon+1}{2 \epsilon+n^{2}}\right) \\
& \times\left[\frac{3}{5}\left(\frac{n^{2}+2}{3}\right)\left(\frac{2 \epsilon+1}{2 \epsilon+n^{2}}\right) \beta^{2} \mu^{02} \Delta \alpha_{\omega}\right. \\
&\left.+\left(\frac{(\epsilon+2)(2 \epsilon+1)}{9 \epsilon}\right) 2 \beta \bar{\beta}_{\omega} \mu^{0}\right] .
\end{aligned}
$$

The only difference is seen to be in the hyperpolarizability term-our $\bar{\beta}_{\omega}$ term must be multiplied by a factor $R=(\epsilon+2)(2 \epsilon+1) / 9 \epsilon$ in order to agree with the corresponding term in $K_{B R}$. But since $R$ is just the ratio of the Lorentz local field to the Onsager cavity field and since we have explicitly used the Onsager cavity field in this term in order to estimate $\bar{\beta}_{\omega e}$, it is clear that the approximations of Buckingham and Raab are equivalent to the use of the Lorentz local field in the $\overline{\boldsymbol{\beta}}_{\omega}$ term. The Lorentz local field, of course, is known to be somewhat less than adequate to the description of polar liquids. ${ }^{1,7}$ The ultimate comparison of (41) and (41') must of course be based upon experiment; the absence of reliable data precludes such a comparison at present.

\section{B. Superposition Approximation}

Equation (38) can be simplified somewhat by assuming that whenever dipoles 2 and 3 are substantially correlated, the direction of dipole 1 is uncorrelated with them. Then averaging over the orientations of dipole 1 gives $\left\langle\cos \gamma_{12} \cos \gamma_{13}\right\rangle_{\infty}=(1 / 3)\left\langle\cos \gamma_{12}\right\rangle_{\infty}$, so that (38) becomes

$$
\begin{aligned}
& \left\langle P_{2}\left(\cos \theta_{1}\right)\right\rangle_{E} / E_{m}^{2}=\frac{1}{15}\left(\beta \mu_{e}\right)^{2}[3 \epsilon /(2 \epsilon+1)]^{2} \\
& \quad \times\left[1+(N-1)\left\langle P_{2}\left(\cos \gamma_{12}\right)\right\rangle_{\infty}+2(N-1)\left\langle\cos \gamma_{12}\right\rangle_{\infty}\right] .
\end{aligned}
$$

Now only the two-particle local distribution function is required.

\section{Weakly Correlated Clusters}

We now consider a model which explicitly reveals the effects of strong short-range anisotropic forces and the short-range correlations which they produce. The $N$ dipoles of the fluid are assumed to "condense" into $n$ identical stable, rigid $s$ mers $(N=s n)$. Some indication of the cluster structure that one might wish to adopt for a particular system might be obtained from the structure of the solid. Such an approach was taken by Kirkwood ${ }^{1}$ to estimate the dielectric constant of water. Each cluster is assumed to have a net dipole moment, denoted for a representative cluster 1 by $\hat{\mu}_{1}$, that interacts with the external field and other clusters according to the theory described previously. Then we can write, from (36) and (38),

$$
\left\langle\cos \theta_{1 R}\right\rangle_{E} / E_{m}=\frac{1}{3} \beta \hat{\mu}[3 \epsilon /(2 \epsilon+1)]\left[1+(n-1)\left\langle\cos \hat{\gamma}_{12}\right\rangle_{\infty}{ }^{\prime}\right] \text {, }
$$

$$
\begin{aligned}
& \left\langle P_{2}\left(\cos \theta_{1 R}\right)\right\rangle_{E} / E_{m}{ }^{2}=\frac{1}{15}(\beta \hat{\mu})^{2}\left[3 \epsilon /(2 \epsilon+1)^{2}\right] \\
& \times\left[1+(n-1)\left\langle P_{2}\left(\cos \hat{\gamma}_{12}\right)\right\rangle_{\infty}^{\prime}\right. \\
& +\frac{3}{2}(n-1)(n-2)\left\langle\cos \hat{\gamma}_{12} \cos \hat{\gamma}_{13}\right\rangle_{\infty}^{\prime} \\
& \left.\quad-\frac{1}{2}(n-1)(n-6)\left\langle\cos \hat{\gamma}_{12}\right\rangle_{\infty}^{\prime}\right],
\end{aligned}
$$

where $\theta_{1 R}$ refers to the angle between the external electric field and the net dipole moment of cluster 1 , $\hat{\gamma}_{i j}$ refers to the angle between the net dipole moments of clusters $i$ and $j$, and the primed brackets indicate that the average is over the positions and orientations of the clusters only. If we call $\alpha_{i}$ the angle in a clusterfixed frame between $\hat{\mu}$ and a constituent dipole $\boldsymbol{\mu}_{i}$, 
TABLE II. Polarization and alignment for certain rigid noninteracting clusters. ${ }^{\mathrm{a}}$

\begin{tabular}{|c|c|c|c|}
\hline Model & $\left(\left\langle\cos \theta_{1}\right\rangle_{E} / \frac{1}{3} \beta \hat{\mu} E_{0}\right)$ & {$\left[\left\langle P_{2}\left(\cos \theta_{1}\right)\right\rangle_{E} / \frac{1}{15}\left(\beta \hat{\mu} E_{0}\right)^{2}\right]$} & $\left(\eta / \frac{3}{5}\right)$ \\
\hline 1. $s$ parallel dipoles $\uparrow \uparrow \cdots \cdots \uparrow$ & $s$ & $s^{2}$ & 1 \\
\hline $\begin{array}{l}\text { 2. } s \text { alternating dipoles } \\
\uparrow \downarrow \uparrow \cdots(s \text { odd })\end{array}$ & $1 / s$ & 1 & $s^{2}$ \\
\hline 3. Case 2 but $s$ even & 0 & 0 & (3) \\
\hline $\begin{array}{l}\text { 4. Umbrella with one stem and } \\
(s-1) \text { equidistant spokes } \\
\text { perpendicular to it. } s>3\end{array}$ & $1 / s$ & $\frac{1}{2}[(3-s) / s]$ & $\frac{1}{2} s(3-s)$ \\
\hline
\end{tabular}

${ }^{a} E_{0} \equiv 3 \epsilon E_{m} /(2 \epsilon+1)$.

application of the addition theorem of spherical harmonics and the symmetry of the problem yields for the two quantities of interest

$$
\begin{aligned}
\left\langle\cos \theta_{1}\right\rangle_{E} & =\left\langle\cos \theta_{1 R}\right\rangle_{E} S^{-1} \sum_{i=1}^{s} \cos \alpha_{i}, \\
\left\langle P_{2}\left(\cos \theta_{1}\right)\right\rangle_{E} & =\left\langle P_{2}\left(\cos \theta_{1 R}\right)\right\rangle_{E} S^{-1} \sum_{i=1}^{s} P_{2}\left(\cos \alpha_{i}\right) .
\end{aligned}
$$

Consequently, within the framework of this model of the liquid, we have a prescription for finding $\left\langle\cos \theta_{1}\right\rangle_{E}$ and $\left\langle P_{2}\left(\cos \theta_{1}\right)\right\rangle_{E}$, given a knowledge of the structure of the cluster and of the short-range angular correlations between different clusters. Note that the intermolecular forces play an important role in determining both of these factors.

If we assume the correlation between different clusters to be negligible, (42) and (43) become

$$
\begin{gathered}
\left\langle\cos \theta_{1 R}\right\rangle_{E} / E_{m}=\frac{1}{3} \beta \hat{\mu}[3 \epsilon /(2 \epsilon+1)], \\
\left\langle P_{2}\left(\cos \theta_{1 R}\right)\right\rangle_{E} / E_{m}{ }^{2}=\frac{1}{15}(\beta \hat{\mu})^{2}[3 \epsilon /(2 \epsilon+1)]^{2},
\end{gathered}
$$

and $\eta$ becomes

$$
\eta=\left(\frac{3}{5} s\right)\left[\sum_{i=1}^{s} P_{2}\left(\cos \alpha_{i}\right) /\left(\sum_{i=1}^{s} \cos \alpha_{i}\right)^{2}\right] .
$$

In Table II we give the polarization and alignment calculated for various special types of clusters under the assumption of negligible correlation between clusters. We also give the values of $\eta$ which result. We note that while the polarization does not distinguish, for example, between the arrangements of cases 2 and 4 , the alignment does. It is clear that the experimentally accessible quantity $\eta$ is quite sensitive to the type of local order which is present.

In this section we have restricted our attention to the perhaps artificial situation in which a single type of rigid cluster is important. It would be quite possible to generalize this approach to a fluid consisting of a mixture of clusters of various sizes and kinds in chemical equilibrium with one another. At the expense of introducing new adjustable parameters, one could then no doubt fit any experimental measurements into the framework of the present theory. However, the crudity of the cluster model is such that a detailed examination of this idea does not seem justified.

\section{IMPERFECT GASES AND RELATED GENERAL DISCUSSION}

It is well known that the two- and three-particle distribution functions can be simply related to pairwise intermolecular potentials through a density expansion. ${ }^{33,34}$ In fact, measurements of the second virial coefficients of the dielectric and Kerr constants can and do yield useful information about anisotropic intermolecular potentials. ${ }^{35} \mathrm{We}$ will not perform density expansions of the results in this paper, since rigorous theories of the dielectric and Kerr constants have been written ${ }^{20,36-38}$ and these are the proper starting place for density expansions. Being rigorous, they lead to exact expressions for the virial coefficients, while our expressions, being approximate, would yield approximate virial coefficients.

The difference in philosophy between our treatment and more rigorous ones is that our treatment has been mainly oriented toward application to liquids, and to this end we have neglected fluctuations in the effective molecular dipole moment, polarizability, and hyperpolarizability at several key points in our derivations of expressions for the dielectric and Kerr constants. Our motivation in doing this was to reveal the dependence of these quantities upon the quantities $\left\langle P_{n}\left(\cos \theta_{1}\right)\right\rangle_{E}$. One intuitively feels that such a description must be possible, at least to some degree of approximation since one feels that the primary physical process behind the Kerr effect and dielectric polarization is the lining up of single molecules with the electric field. When this idea is pursued, it is found that the equations take a pleasingly intuitive form-the effects can indeed be regarded as the lining up of single molecules possessing effective permanent moments, polarizability anisotropies, etc., given by well-defined statistical expressions. This sort of physical insight is for the most part lacking in the results of the more formal rigorous theories, which are not readily simplified to reveal the roles of the polarization and alignment and which may not be applied to liquids without drastic assumptions anyway. In the case of slightly imperfect gases, however, the 
complexity of the rigorous theories decreases to a manageable level, so that rigorous results for the virial coefficients can be obtained from them; there is therefore no need to neglect fluctuations and no reason to do so.

We remark that our results for the dielectric and Kerr constants, derived in Secs. II and IV, can be obtained from a rigorous theory which provides explicit expressions for the fluctuation terms which we have neglected. This theory will be the subject of a future communication. We also note that it has not been necessary to specify a molecular model (such as a conventional permanent moment plus isotropic harmonic-oscillator polarizability) at the outset in order to make progress. For example, the expression (4a) for the effective moment is quite general, and makes no reference to the existence of the various parameters describing the distortion of molecule 1 by its neighbors. The full complexity of this distortion is retained until such time as one attempts to evaluate the expression for $\mu_{e}$.

\section{APPENDIX A: ISOLATED MOLECULE IN A UNIFORM FIELD}

We here review the conventional description and terminology of the interaction between an isolated molecule and a uniform external electric field. ${ }^{2}$ The dipole moment of the molecule, $\boldsymbol{\mu}$, may be expanded in powers of the applied field $\mathbf{E}$ :

$$
\boldsymbol{\mu}=\boldsymbol{\mu}^{0}+\mathbf{a} \cdot \mathbf{E}+\frac{1}{2} B: \mathbf{E E}+\cdots .
$$

Here $\mu^{0}$ is the permanent moment of the molecule, $\boldsymbol{a}$ is the (ordinary) polarizability, B is the first hyperpolarizability, and so on. These quantities are respectivaly tensors of rank one, two, three, and so on. These tensors may be shown to be symmetric. They may readily be expressed as derivatives of $\mu$ with respect to E:

$$
\begin{gathered}
\boldsymbol{\mu}^{0}=\left.\boldsymbol{\mu}\right|_{E=0} ; \\
\mathbf{a}=(\partial \mu / \partial \mathbf{E})_{E=0} ; \quad \mathrm{B}=\left(\partial^{2} \boldsymbol{\mu} / \partial \mathbf{E} \partial \mathbf{E}\right)_{E=0} ; \text { etc. }
\end{gathered}
$$

In a polar liquid, the field acting on a molecule due to its neighbors is much stronger than typical laboratory electric fields, so that the customary linear polarization law is not adequate. Moments are also induced by field gradients and by short-range molecular interactions, so the situation in an actual polar liquid is very complex. In general, the fields due to nearby molecules are not so strong that terms higher than the quadratic need to be retained in (A1), although each case should be separately investigated. The B term, however, is often important.

\section{APPENDIX B : REMOVAL OF BOUNDARY EFFECTS}

Consider a large macroscopic spherical sample (of radius $b$ ) in vacuum and divide its interior into two regions: Region 1 is the volume within a smaller but still macroscopic sphere (of radius $a$ ) centered within the large sphere, and region 2 is the volume exterior to the small sphere. We assume that molecules are not allowed to pass from one region to the other and that the number density is the same in both regions. Molecule 1 is chosen to be in region 1 . No loss of generality is incurred by these restrictions. The limit $b / a \rightarrow \infty$ is ultimately to be taken; in this limit, region 1 can be considered to be a sphere immersed in an infinite medium of the same sample material.

The configuration of the total system is $\left(\tau_{1}, \tau_{2}\right)$, where $\tau_{i}$ is the configuration of the molecules in region $i$. The total moment $\mathbf{M}$ of the system is the sum of the moments of the two regions: $\mathbf{M}=\mathbf{M}_{1}+\mathbf{M}_{2}$. Because of our replacement of each molecular moment by an effective permanent moment, the moment of region $i$, $\mathbf{M}_{\mathbf{i}}$, depends only upon $\tau_{\mathrm{i}}$. Then we have

$$
\left\langle\mathbf{e}_{1} \cdot \mathbf{M}\right\rangle_{0}=\left\langle\mathbf{e}_{1} \cdot\left(\mathbf{M}_{1}+\mathbf{M}_{2}\right)\right\rangle_{0} .
$$

Clearly $\left\langle\mathbf{e}_{1} \cdot \mathbf{M}_{\mathbf{1}}\right\rangle_{0}=\left\langle\mathbf{e}_{\mathbf{1}} \cdot \mathbf{M}\right\rangle_{\infty}$. Thus we need only consider $\left\langle\mathbf{e}_{1} \cdot \mathbf{M}_{2}\right\rangle_{0}$. Let $f\left(\tau_{1}, \tau_{2}\right) d \tau_{1} d \tau_{2}$ be the probability that the configuration of the system is in $d \tau_{1} d \tau_{2}$ at $\left(\tau_{1}, \tau_{2}\right)$. By the fundamental theorem of conditional probability, we have

$$
f\left(\tau_{1}, \tau_{2}\right)=f\left(\tau_{1}\right) f\left(\tau_{1} \mid \tau_{2}\right),
$$

where $f\left(\tau_{1}\right) d \tau_{1}$ is the probability that the molecules of region 1 are in $d \tau_{1}$ at $\tau_{1}$ regardless of the configuration of the molecules of region 2 , and $f\left(\tau_{1} \mid \tau_{2}\right) d \tau_{2}$ is the probability that the molecules of region 2 are in $d \tau_{2}$ at $\tau_{2}$ given that the molecules of region 1 are in $d \tau_{1}$ at $\tau_{1}$. Then

$$
\left\langle\mathbf{e}_{1} \cdot \mathbf{M}_{2}\right\rangle_{0}=\int d \tau_{1} f\left(\tau_{1}\right) \mathbf{e}_{1} \cdot \int d \tau_{2} f\left(\tau_{1} \mid \tau_{2}\right) \mathbf{M}_{2}\left(\tau_{2}\right) .
$$

But $\int d \tau_{2} f\left(\tau_{1} \mid \tau_{2}\right) \mathbf{M}_{2}\left(\tau_{2}\right)$ is the mean moment of region 2 for fixed $\tau_{1}$. Fixing $\tau_{1}$ also fixes the field with which region 2 interacts. Therefore $\int d \tau_{2} f\left(\tau_{1} \mid \tau_{2}\right) \mathbf{M}_{2}\left(\tau_{2}\right)$ is merely the statistical average of the macroscopic moment $\mathbf{M}_{2}$ of region 2 in a fixed external field, namely that produced by the molecules of region 1 at fixed $\tau_{1}$. The point to appreciate now is that this quantity may be calculated by the use of macroscopic electrostatics (that is, by treating region 2 as a continuum of dielectric constant $\epsilon$ ) since macroscopic averages obey the macroscopic equations. The result of a straightforward electrostatic calculation similar to that of Kirkwood $^{1}$ is that

$$
\int d \tau_{2} f\left(\tau_{1} \mid \tau_{2}\right) \mathbf{M}_{2}\left(\tau_{2}\right)=c(\epsilon) \mathbf{M}_{1}\left(\tau_{1}\right)
$$

in the limit $b \gg a$, where $1+c(\epsilon)=9 \epsilon /[(2 \epsilon+1)(\epsilon+2)]$. Implicit in the above is the assumption that the field due to the molecules in region 1 is not large enough to produce nonlinear behavior (i.e., dielectric saturation) in region 2. This condition is satisfied except for rare configurations $\tau_{\mathbf{1}}$. 
Combining the above results yields

$$
\left\langle\mathbf{e}_{1} \cdot \mathbf{M}\right\rangle_{0}=\left\{\mathbf{g}_{\epsilon} /[(2 \epsilon+1)(\epsilon+2)]\right\}\left\langle\mathbf{e}_{\mathbf{1}} \cdot \mathbf{M}\right\rangle_{\infty},
$$

which is identical with (35).

We must now carry out the above procedure for the average $\left\langle P_{2}\left(\mathbf{e}_{1} \cdot \mathbf{e}_{\|}\right)\left(\mathbf{M} \cdot \mathbf{e}_{\| \mid}\right)^{2}\right\rangle_{0}$. We have

$$
\begin{aligned}
& \left\langle P_{2}\left(\mathbf{e}_{1} \cdot \mathbf{e}_{\| \mid}\right)\left(\mathbf{M} \cdot \mathbf{e}_{\| \mid}\right)^{2}\right\rangle_{0}=\left\langle P_{2}\left(\mathbf{e}_{1} \cdot \mathbf{e}_{\| \mid}\right)\left(\mathbf{M}_{1} \cdot \mathbf{e}_{\| \mid}\right)^{2}\right\rangle_{0} \\
& +2\left\langle P_{2}\left(\mathbf{e}_{1} \cdot \mathbf{e}_{\| \mid}\right)\left(M_{1} \cdot \mathbf{e}_{\| \mid}\right)\left(\mathbf{M}_{2} \cdot \mathbf{e}_{\| 1}\right)\right\rangle_{0} \\
& +\left\langle P_{2}\left(\mathbf{e}_{1} \cdot \mathbf{e}_{\| I}\right)\left(\mathbf{M}_{2} \cdot \mathbf{e}_{\| \mid}\right)^{2}\right\rangle_{0} .
\end{aligned}
$$

Clearly $\left\langle P_{2}\left(\mathbf{e}_{1} \cdot \mathbf{e}_{\| 1}\right)\left(\mathbf{M}_{1} \cdot \mathbf{e}_{11}\right)^{2}\right\rangle_{0}=\left\langle P_{2}\left(\mathbf{e}_{1} \cdot \mathbf{e}_{\| 1}\right)\left(\mathbf{M} \cdot \mathbf{e}_{11}\right)^{2}\right\rangle_{\infty}$, and a trivial reapplication of the arguments leading to (B1) yields

$$
\begin{aligned}
& \left\langle P_{\mathbf{2}}\left(\mathbf{e}_{1} \cdot \mathbf{e}_{\|}\right)\left(\mathbf{M}_{1} \cdot \mathbf{e}_{\| \mid}\right)\left(\mathbf{M}_{2} \cdot \mathbf{e}_{\| I}\right)\right\rangle_{0} \\
& =c(\boldsymbol{\epsilon})\left\langle P_{2}\left(\mathbf{e}_{1} \cdot \mathbf{e}_{\|}\right)\left(\mathbf{M} \cdot \mathbf{e}_{\| \mid}\right)^{2}\right\rangle_{\infty} .
\end{aligned}
$$

We therefore need only to consider $\left\langle P_{2}\left(\mathbf{e}_{1} \cdot \mathbf{e}_{\| \mid}\right) \times\right.$ $\left.\left(\mathbf{M}_{2} \cdot \mathbf{e}_{\mid 1}\right)^{2}\right\rangle_{0}$ :

$$
\begin{aligned}
\left\langle P_{2}\left(\mathbf{e}_{1} \cdot \mathbf{e}_{\| \mid}\right)\left(\mathbf{M}_{2} \cdot \mathbf{e}_{\| \mid}\right)^{2}\right\rangle_{0}= & \int d \tau_{1} f\left(\tau_{1}\right) P_{2}\left(\mathbf{e}_{1} \cdot \mathbf{e}_{\| \mid}\right) \\
& \times \int d \tau_{2} f\left(\tau_{1} \mid \tau_{2}\right)\left[\mathbf{M}_{2}\left(\tau_{2}\right) \cdot \mathbf{e}_{\| I}\right]^{2} .
\end{aligned}
$$

By considering the linear response of region 2, in the presence of region 1 at fixed $\tau_{1}$, to an externally applied field $\mathbf{E}$, it is possible to show that

$$
\begin{aligned}
\int d \tau_{2} f\left(\tau_{1} \mid \tau_{2}\right)\left[\mathbf{M}_{2}\left(\tau_{2}\right) \cdot \mathbf{e}_{\| !}\right]^{2} & -\left[\int d \tau_{2} f\left(\tau_{1} \mid \tau_{2}\right) \mathbf{M}_{2}\left(\tau_{2}\right) \cdot \mathbf{e}_{\| \mid}\right]^{2} \\
= & \beta^{-1}\left[\partial \mathbf{M}_{2}^{\prime}(\mathbf{E}) / \partial \mathbf{E}\right]_{E=0}: \mathbf{e}_{\| \mid} \mathbf{e}_{\| \mid},
\end{aligned}
$$

where $\mathbf{M}_{2}^{\prime}(\mathbf{E})$ is the mean moment which region 2 would have in the presence of $\mathbf{E}$ if the molecules of region 1 were removed; that is, if region 1 were an empty cavity of radius $a$. The right-hand side of the above is therefore independent of $\tau_{1}$. Since

we then have

$$
\int d \tau_{1} f\left(\tau_{1}\right) P_{2}\left(\mathbf{e}_{1} \cdot \mathbf{e}_{\mid 1}\right)=0,
$$

$$
\left\langle P_{2}\left(\mathbf{e}_{1} \cdot \mathbf{e}_{\|}\right)\left(\mathbf{M}_{2} \cdot \mathbf{e}_{\| I}\right)^{2}\right\rangle_{0}=c^{2}(\boldsymbol{\epsilon})\left\langle P_{2}\left(\mathbf{e}_{1} \cdot \mathbf{e}_{\| I}\right)\left(\mathbf{M} \cdot \mathbf{e}_{\| \mid}\right)^{2}\right\rangle_{\infty} \text {. }
$$

We have again made the implicit assumption that the field due to the molecules in region 1 is not large enough to produce dielectric saturation in region 2. Our final result is

$$
\begin{aligned}
\left\langle P_{2}\left(\mathbf{e}_{1} \cdot \mathbf{e}_{\| 1}\right)\left(\mathbf{M} \cdot \mathbf{e}_{\mid 1}\right)^{2}\right\rangle_{0}= & {\left[1+2 c(\epsilon)+c^{2}(\boldsymbol{\epsilon})\right] } \\
& \times\left\langle P_{2}\left(\mathbf{e}_{1} \cdot \mathbf{e}_{\| \mid}\right)\left(\mathbf{M} \cdot \mathbf{e}_{||}\right)^{2}\right\rangle_{\infty} \\
= & \{9 \epsilon /[(2 \epsilon+1)(\epsilon+2)]\}^{2} \\
& \times\left\langle P_{2}\left(\mathbf{e}_{1} \cdot \mathbf{e}_{\| \mid}\right)\left(\mathbf{M} \cdot \mathbf{e}_{\| \mid}\right)^{2}\right\rangle_{\infty} . \quad(\mathbf{B} 2)
\end{aligned}
$$

We emphasize that our treatment has been a molecular one, since both the small sphere and its surroundings were treated from the beginning on a molecular basis. However, it is clear that the above developments could not have been carried out without using the fact that the moment of region $i, \mathbf{M}_{i}$, depends only on $\tau_{i}$, which was a consequence of our replacement of molecular dipole moments by effective permanent moments. If this approximation is not made and the molecules are polarizable, then both $\mathbf{M}_{1}$ and $\mathbf{M}_{\mathbf{2}}$ depend on both $\tau_{1}$ and $\tau_{2}$. In such a case, it may be a useful approximation merely to replace the molecules in region 2 by a true continuum of dielectric constant $\epsilon$, even though it is unclear how to justify such a procedure on a molecular basis. The above development justifies this procedure for the case of rigid dipoles, for in that case $\mathbf{M}_{i}$ depends only on $\tau_{i}$; this was pointed out by Buckingham. ${ }^{39}$ In the case of polarizable dipoles (which are all that exist in the real world) the precise nature of this "continuum" approximation and the limits of its validity are not at present known.

* This work was supported in part by the National Science Foundation.

$\dagger$ NSF Predoctoral Fellow.

$\ddagger$ Present address: Department of Physics, Massachusetts Institute of Technology, Cambridge, Mass.

$\$$ Alfred P. Sloan Research Fellow. Address after January 1970: Department of Chemistry, Massachusetts Institute of Technology, Cambridge, Mass.

1 J. G. Kirkwood, J. Chem. Phys. 7, 911 (1939).

2 A. R. Edmonds, Angular Momentum in Quantum Mechanics (Princeton U. P., Princeton, N.J., 1960).

${ }^{3}$ A similar point of view has been outlined in a brief survey paper by A. D. Buckingham, Discussions Faraday Soc. 43, 205 (1967).

${ }^{4}$ L. Onsager, J. Am. Chem. Soc. 58, 1486 (1936)

${ }^{5}$ F. E. Harris and B. J. Alder, J. Chem. Phys. 21, 1031 (1953).

6 F. E. Harris, J. Chem. Phys. 23, 1663 (1955).

${ }^{7}$ W. F. Brown, Jr., Handbuch der Physik, edited by S. Flugge (Springer, Berlin, 1956), Vol. 17, p. 1.

${ }^{8}$ A. Abragam, Principles of Nuclear Magnetism (Oxford U. P., Oxford, London, 1961), Chap. 8.

${ }^{9}$ E. L. Hahn, Phys. Rev. 93, 944 (1954).

${ }_{10}$ M. W. P. Strandberg, Phys. Rev. 127, 1162 (1962).

"A. D. Buckingham and E. G. Lovering, Trans. Faraday Soc. 58, 2077 (1962).

${ }_{12}$ A. M. Vasil'ev, Zh. Eksp. Teor. Fiz. 43, 1526 (1962) [Sov. Phys. JETP 16, 1078 (1963)].

${ }^{13} \mathrm{~F}$. Mannis, Ph.D. thesis, Massachusetts Institute of Technology, Cambridge, Mass., 1962.

${ }_{14}$ C. W. Hilbers and C. MacLean, Mol. Phys. 16, 275 (1969); 17, 433, 517 (1969).

ib J. S. Waugh, Lectures on Solid State Physics, Hercegnovi 1963 (Federal Nuclear Energy Commission of Yugoslavia, Ljubljana, 1964), p. 93.

${ }_{16}$ P. Debye, Polar Molecules (Chemical Catalog, New York, 1929), Chaps. 1,3

${ }^{17}$ Of course, one could write a rigorous formal statisticalmechanical expression for the average local field seen by a representative molecule. However, the use of such an expression for $\mathbf{F}$ would still be an approximation, since the molecule does not interact at every instant with an average local field, but rather with the instantaneous local field. It is the average of this instantaneous interaction, not the average field, which one measures.

${ }^{18} \mathrm{H}$. Goldstein, Classical Mechanics (Addison-Wesley, Reading, Mass., 1950), Chaps. 4, 5.

${ }_{19}$ A. D. Buckingham and K. McLauchlan, Proc. Chem. Soc. 1963,144

${ }^{20}$ A. D. Buckingham and R. E. Raab, J. Chem. Soc. 1957, 2341.

${ }_{21}$ A. D. Buckingham and J. A. Pople, Proc. Phys. Soc. (London) 68, 905 (1955)

${ }^{22}$ C. J. F. Böttcher, Theory of Electric Polarisation (Elsevier, Amsterdam, 1952).

${ }^{23}$ C. G. LeFèvre and R. J. W. LeFèvre, Technique of Organic Chemistry, edited by A. Weissberger (Interscience, New York, 1960), 3rd ed., Vol. 1, Pt. III, p. 2459.

${ }_{24}$ S. Kielich, Acta Phys. Polon. 22, 299 (1962). 
${ }^{25}$ A. D. Buckingham and B. J. Orr, Quart. Rev. (London) 21, 195 (1967).

26 J. M. O'Hare and R. P. Hurst, J. Chem. Phys. 46, 2356 (1967).

${ }^{27}$ R. J. W. LeFèvre and B. P. Rao, J. Chem. Soc. 1958, 1465.

${ }^{28}$ C. G. LeFèvre and R. J. W. LeFèvre, Rev. Pure Appl. Chem. 5,261 (1955).

${ }^{29}$ A. D. B uckingham and M. J. Stephen, Trans. Faraday Soc. 53,884 (1957).

${ }^{30}$ This assumption has been universally made, especially in the case of the dielectric constant. However, its content is nontrivial and in our opinion the question of its validity has not received sufficient attention. In the case of the dielectric constant, the assumption is equivalent to the assumption that the ratio $\mathbf{P}(\mathbf{r}) / \mathbf{E}_{m}(\mathbf{r})$ is a constant of the material, independent of $\mathbf{r}$ and the same for any sample geometry. An answer to the question of its validity would necessarily require the consideration of an arbitrary sample geometry in which $\mathbf{P}$ and $\mathbf{E}_{m}$ might well be nonuniform since one has no way of knowing a prior $i$ whether $\mathbf{P}$ and $\mathbf{E}_{m}$ are uniform for any particular geometry. We are currently considering this problem.

31 The two-particle angle-dependent distribution function suffices for the calculation of the polarization, while both the twoand three-particle functions are needed to calculate the alignment.
Of course, this situation would be greatly complicated if we had not replaced each molecular dipole moment by an effective permanent moment; for polarizable dipoles the distribution functions of all orders are theoretically required (see Ref. 36). In practice, a power series in the molecular polarizability and other parameters could be written and only a few low-order distribution functions would probably be required, but the situation would still be vastly more complex than it is now.

${ }^{32} \mathrm{~J}$. M. Deutch and J. S. Waugh, J. Chem. Phys. 43, 2568 (1965) ; 44, 4366 (1966).

${ }_{33}$ P. Mazur and I. Oppenheim, Physica 23, 216 (1957).

${ }^{34}$ L. Van Kampen, Physica 27, 783 (1961)

${ }^{35}$ See, for example, H. Sutter and R. H. Cole, J. Chem. Phys. 46, 2014 (1967) ; H. B. Levine and D. A. McQuarrie, ibid. 44, 3500 (1966); A. D. Buckingham, Proc. Phys. Soc. (London) 68, 910 (1955); D. W. Schaefer, Ph.D. thesis, Massachusetts Institute of Technology, 1968, and references cited therein.

${ }^{36} \mathrm{M}$. Mandel and P. Mazur, Physica 24, 116 (1958).

${ }^{37}$ A. D. Buckingham and J. A. Pople, Trans. Faraday Soc. $51,1029(1955)$.

${ }_{38}$ P. Mazur and B. J. Postma, Physica 25, 251 (1959).

${ }^{39}$ A. D. Buckingham, Proc. Roy. Soc. (London) A238, 235 (1956).

\title{
Light Scattering from Chemically Reactive Fluids. IV. Intensity Calculations for the Eulerian Fluid with One Reaction*
}

\author{
Dwayne L. Knirk $†$ AND Z. W. SALsburg \\ Department of Chemistry, Rice University, Houston, Texas 77001
}

(Received 23 September 1970)

\begin{abstract}
The hydrodynamic equations for the reactive Eulerian fluid (zero transport coefficients, one chemical reaction) have been used in the thermodynamic approach to calculate in detail the spectrum of scattered light. The mathematical technique employed was the matrix eigenvalue formulation previously introduced [L. Blum and Z. W. Salsburg, J. Chem. Phys. 48, 2292 (1968) ]. The method focuses on a particular matrix which is easily derived from the linearized hydrodynamic equations. The fluctuations of the set of independent variables are resolved into normal modes of relaxation. Each mode contributes one peak to the spectrum; the position and half-width of the peak are furnished directly by the eigenvalue, while the intensity is calculated from the corresponding normal mode projection matrix. Some general relationships between positions and half-widths are derived. The Rayleigh peak due primarily to chemical reaction relaxation is considered in detail. Various intensity ratios involving this peak are calculated, and simple criteria are set forth for determining whether it will be intense enough to be experimentally observed. If it is observable, the reaction rate constant can be obtained from measurements of its half-width. Two other chemical relaxation effects, dispersion of the sound speed and skewing of the Brillouin peaks, are also examined. For small scattering angles and very fast reactions, the rate constant may be extracted from measurements of the sound speed dispersion. The range of relaxation times for which experiments seem feasible is $10^{-4-10^{-11}} \mathrm{sec}$.
\end{abstract}

\section{INTRODUCTION}

In recent years a number of articles have been written on the theory of the spectral distribution of light scattered from a chemically reactive fluid. ${ }^{1-7}$ The theoretical work on this problem has been much more extensive than the experimental work, ${ }^{8,9}$ partially because the theoretical papers have offered little guidance for the experimentalists.

Some of the analyses ${ }^{1,3,8,9}$ have been limited in scope and are primarily elementary model calculations which illustrate the major effects of the chemical reaction processes. These calculations tend to ignore hydrodynamic fluctuation effects. The more general papers $^{2,4,5,7}$ are quite formal in nature and present the results in such abstract form that it is almost impossible to apply them.

The complete hydrodynamic fluctuation analysis, which forms the core of these phenomenological theories of light scattering, is rather involved and leads to complicated expressions describing the intensity of the scattered light. One of the challenges which still remains is to carry the analysis to the point where one can make detailed calculations for real chemical systems and at the same time give a presentation which is comprehensible by both the experimentalist and the theoretician.

This article is intended as a step toward accomplish- 